\title{
Population-based body-brain mapping links brain morphology and body composition
}

Authors: Tiril P Gurholt, $\mathrm{PhD}^{1,{ }^{*}}$, Tobias Kaufmann, $\mathrm{PhD}^{1}$, Oleksandr Frei, $\mathrm{PhD}^{1}$, Dag Alnæs, $\mathrm{PhD}^{1}$, Unn K Haukvik, $\mathrm{PhD}^{1}$, Dennis van der Meer, $\mathrm{PhD}^{1,2}$, Torgeir Moberget, $\mathrm{PhD}^{1,3}$, Kevin S O’Connell, $\mathrm{PhD}^{1}$, Olof D Leinhard, $\mathrm{PhD}^{4,5}$, Jennifer Linge, $\mathrm{MSc}^{4}$, Rozalyn Simon, $\mathrm{PhD}{ }^{4,5}$, Olav B Smeland, $\mathrm{PhD}^{1}$, Ida E Sønderby, $\mathrm{PhD}^{1,6}$, Adriano Winterton, MD ${ }^{1}$, Nils Eiel Steen, $\mathrm{PhD}^{1}$, Lars T Westlye, $\mathrm{PhD}^{1,3}$, Ole A Andreassen, $\mathrm{PhD}^{1}$.

\section{Affiliations}

${ }^{1}$ Norwegian Centre for Mental Disorders Research (NORMENT), Division of Mental Health and Addiction, Oslo University Hospital and University of Oslo, Oslo, Norway

${ }^{2}$ School of Mental Health and Neuroscience, Faculty of Health, Medicine and Life Sciences, Maastricht, The Netherlands

${ }^{3}$ Department of Psychology, University of Oslo, Oslo, Norway

${ }^{4}$ AMRA Medical, Linköping, Sweden

${ }^{5}$ Division of Diagnostics and Specialist Medicine, Department of Health, Medicine and Caring Sciences, Linköping University, Linköping, Sweden

${ }^{6}$ Department of Medical Genetics, Oslo University Hospital, Oslo, Norway

* Corresponding author: Tiril P Gurholt, PhD. E-mail: t.p.gurholt@medisin.uio.no. Postal address: Oslo University Hospital, P.O.Box 4956 Nydalen, 0424 OSLO, Norway, phone: +47 977627 76, fax: +4723027333.

Keywords: Brain structure; Magnetic resonance imaging; Adiposity; Muscle volume; Anthropometric measures; Body MRI; Healthy population. 


\begin{abstract}
Background: Understanding complex body-brain processes, and putative interplay between adipose tissue and brain health, is of vital importance for brain and somatic disease prevention in the general population. We studied the link between body composition and brain structure through large-scale investigation in a healthy population without secondary disease effects.
\end{abstract}

Methods: We processed brain magnetic resonance imaging (MRI) data and extracted measures of brain morphometry from 19,330 healthy UK Biobank participants, of which a subset $(n=2,703)$ had body MRI. We investigated associations between brain structure and (i) anthropometric body composition measures, and (ii) regional/specific body MRI measures of abdominal fat and muscle tissue.

Findings: We identified highly significant body-brain associations ( $p$-values $\leq 0 \cdot 0002$ ). Anthropometric measures showed negative, nonlinear, associations with cerebellar/cortical gray matter, and brain stem structures, negative associations with white matter, and positive associations with ventricular volumes. Subcortical structures exhibited mixed effect directionality, with strongest positive association for accumbens. Among body MRI measures, liver fat was negatively associated with thinner/lower cortical gray matter thickness/volume, and thigh muscle volume positively associated with accumbens volume.

Interpretation: We demonstrate significant body-brain associations, and map individual differences in body composition to brain morphology in healthy individuals. Common measures of body composition correlated negatively with cerebellar and cortical structures and positively with the accumbens, a dopamine rich structure involved in reward processing. These findings of a relationship between brain anatomy and body composition provide new insight into body-brain processes and suggest shared mechanisms of cardiometabolic risk factors and brain disorders. This may form the foundation for a new type of prevention studies, and provides a framework for studies of underlying mechanisms related to unhealthy lifestyle and obesity, with implications for public health and prevention.

Funding: The Research Council of Norway, South-Eastern Norway Regional Health Authority, European Union's Horizon 2020 Research and Innovation Programme \& European Research Council. 


\section{Research in context}

\section{Evidence before this study}

Prior studies have indicated an association between brain structure and both obesity and fitness levels - of opposing directionality. Despite this, normal body-brain association patterns in healthy individuals have not been established, and the causal mechanisms are unclear. To enhance our understanding and establish the link between the body and the brain, we saw the need for large-scale investigations in healthy populations. For the study, we searched the PubMed database from March $12^{\text {th }}, 2019$, through February $25^{\text {th }}, 2020$, for scientific literature related to adipose tissue, body composition, brain morphology, and body and brain MRI. Search terms included: body fat, adipose tissue, subcutaneous/visceral adipose tissue, liver fat, body composition, anthropometric measures, body mass index, waist circumference, waist-to-hip ratio, adiposity, obesity, metabolic syndrome, cardiovascular, cardiometabolic, disease/disorder, muscle volume, fitness, brain structure, brain morphology, brain MRI, and body MRI. We based the scientific foundation on review studies, meta-analyses, and other larger studies, but generally excluded smaller studies, and thereby lowering the risk of evidential bias such as winner's curse, although this does not eliminate the risk of publication bias.

\section{Added value of this study}

In the largest study, to date, including 19,330 healthy participants without secondary disease effects, we provide insight into normal body-brain processes by identifying bodybrain associations that map normally varying body composition to brain morphology.

\section{Implications of all the available evidence}

We identified body-brain associations that give insight into normal physiological bodybrain processes in healthy individuals, providing a reference point for studies of underlying mechanisms related to unhealthy lifestyle, obesity, and disorders of the body and the brain. Whereas the directionality and causal chain is unknown, these findings have potential implications for public health and disease prevention. 


\section{Introduction}

Obesity is a risk factor for disorders of both the body ${ }^{1}$ and the brain, ${ }^{1,2}$ and represents a global health challenge. Although causal mechanisms remain unclear, the associations between brain and physical health likely reflect body-brain interactions and common mechanisms across the soma and the mind. Indeed, patients with mental disorders show subtle structural brain alterations as revealed using brain imaging, ${ }^{3}$ and are at increased risk for poor physical health, including obesity, metabolic syndrome, cardiovascular disorders, and shorter life-expectancy. ${ }^{4}$ Yet, how these factors relate to brain health remains poorly understood.

Cross-sectional brain magnetic resonance imaging (MRI) studies have documented negative associations between obesity/poor metabolic health and gray matter volumes ${ }^{5-9}$ and white matter microstructure, ${ }^{5,6}$ while there is conflicting evidence for white matter volume. ${ }^{5,6}$ It is not clear how different aspects of obesity, e.g. adipose tissue distribution, or intra-abdominal fat a known risk factor for adverse health outcomes ${ }^{1,10}$ - relate to brain health. Nonlinear associations are a common phenomenon in neuroimaging (e.g. accelerated brain atrophy at higher ages ${ }^{11}$ ), yet it is unknown whether aspects of obesity are nonlinearly related to brain structure. The genetic contribution to obesity is substantial and interacts with the environment, lifestyle, and sex. ${ }^{12}$ Additionally, body composition/obesity, as well as brain structure, are modifiable and sensitive to environmental and lifestyle factors, and sex. Indeed, physical fitness and activity have been positively associated with gray and white matter volumes ${ }^{13}$, indicating their importance for brain health, while negative associations have been reported for mobility impairment. ${ }^{14}$

Prior studies have largely investigated associations between brain structure and anthropometric measures (e.g. body mass index (BMI), waist-to-hip ratio (WHR)), which are informative, but nonspecific measures of body composition. In contrast, body MRI enables more specific and detailed in vivo measures of regional fat and muscle distribution, allowing for individual body composition profiling which has relevance for clinical prediction. ${ }^{15} \mathrm{How}$, and to what degree, these body MRI measures relate to individual differences in brain structure is unknown.

The pathophysiological mechanisms of psychiatric disorders, and other brain disorders, have proven elusive. To disentangle these complex and multifactorial mechanisms, with each contributing factor having small effects, we need an improved foundational understanding of normal body-brain connections in healthy individuals. To accurately capture such small effects, 
and thereby improve our understanding of normative body-brain connections, large-scale investigations are needed. ${ }^{16}$

To this end, we tested for associations between body composition and brain structure in healthy individuals aged 44 to 82 years using anthropometric measures and brain $(n=19,330)$ and body $(n=2,703)$ MRI from the UK biobank. ${ }^{17}$ Based on prior studies ${ }^{5-9,13}$ we expected brain structure to show negative associations with body composition measures, possibly with stronger associations for intra-abdominal fat, and positive associations with muscle volume. Through large-scale mapping, we hypothesized that we would obtain insight into, and identify robust patterns of normal body-brain connections, in a healthy population.

\section{Methods}

\section{Study design and participants}

We included 19,330 healthy UK biobank participants (10,104 women, 9,226 men) with brain MRI and anthropometric measures (i.e. the full sample). A subsample $(n=2,703)$ had body MRI measures available. We excluded participants with known diagnosis of cancers, selected traumas, neurological, psychiatric, substance abuse, cardiovascular, liver, or severe infectious conditions (Note S1), with incomplete demographic or clinical data, or who withdrew their consent. We did not exclude based on common metabolic syndrome or lifestyle factors, but adjust for these in the analyses.

UK biobank has IRB approval from North West Multi-center Research Ethics Committee and its Ethics Advisory Committee (https://www.ukbiobank.ac.uk/ethics) oversees the UK biobank Ethics \& Governance Framework. ${ }^{17}$ We obtained access to the UK biobank cohort through Application number 27412. The study was approved by the Regional Committees for Medical and Health Research Ethics (https://helseforskning.etikkom.no), and conducted in accordance with the Helsinki Declaration. 


\section{Demographic and clinical data}

We extracted demographic and clinical data (age, sex, ethnicity), and variables reflecting cardiovascular risk (including history of diabetes, hypercholesterolemia, hypertension, current cigarette smoker, current alcohol consumption), and body composition (waist/hip circumference, weight, height). We computed BMI and WHR (Table 1; Note S2).

\section{MRI acquisition}

Participants underwent $3 \mathrm{~T}$ brain and $1.5 \mathrm{~T}$ body/liver MRI on the same day and site. Brain MRI was available from three sites (Cheadle, Reading, and Newcastle), while body/liver MRI was from one site (Cheadle). Similar scanners/protocols were used across all sites ${ }^{17}$ (Note S3).

\section{MRI processing}

We processed brain MRI DICOM images in-house using FreeSurfer (version 5.3.0; http://www.freesurfer.net). We extracted mean cortical thickness and white surface area from the cortical parcellation, and volumes of cortical/cerebellum gray/white matter, brain stem, CSF, lateral ventricle, third ventricle, thalamus, hippocampus, amygdala, accumbens, caudate, putamen, and pallidum. For bilateral measures, we computed the average across the hemispheres. Additionally, we extracted Euler numbers as a proxy of image quality.

We extracted body and liver MRI data processed for abdominal and liver fat, and thigh muscle volumes. Body MRI was processed for visceral adipose tissue (VAT), abdominal subcutaneous adipose tissue (ASAT), total abdominal adipose tissue (VAT+ASAT), and total thigh muscle volume (TTMV) by AMRA (https://www.amramedical.com). Liver MRI was processed for liver proton density fat fraction (PDFF) by Perspectum Diagnostics (https://perspectumdiagnostics.com/) (Note S4). 


\section{MRI quality control}

Among the 33,303 participants with available brain MRI, 21,395 met the inclusion criteria. Of these, we excluded two participants with missing Euler numbers prior to iteratively excluding Euler outliers defined as participants with higher negative Euler numbers that exceeded three standard deviations from the mean in either hemisphere. We iterated until no outliers remained, resulting in eight iterations. This led to further exclusion of 2063 participants, yielding a total sample of n=19,330 (Figure S1; Note S5).

For body MRI, participants labelled with severe motion artifacts, corrupted data, broken coil element, outer field-of-view inhomogeneities, or metal contamination were removed. We further excluded participants with incomplete measures. In total, 857 participants were excluded, largely due to missing liver PDFF measures $(n=676)$, yielding a total body MRI subsample of $n=2,703$ (Figure $\mathrm{S} 1$ ).

\section{Statistical analysis}

We investigated the sample demographics across and within sexes. Categorical variables were compared using $\chi^{2}$-test. For normally/non-normally distributed continuous variables we used the two-sample t-test/Wilcoxon rank-sum test. For unequal variance across sexes, t-test was replaced by Welch approximation. Normality was assessed by visual evaluation of density plots (Figure S2-S3). For brain structure, we assessed density plots for expected distribution patterns (Figure S4-S5), and scatter plots of body-brain associations (Figures S6-S13).

For descriptive purposes, and to establish fundamental properties of the dataset, we initially assessed age- and sex-related associations on body composition and brain structure using a three-step multiple linear regression approach: model $1 \mathrm{a}$ included age, age ${ }^{2}$, and sex; model $1 \mathrm{~b}$ additionally included age-by-sex and age ${ }^{2}$-by-sex interactions; and model $1 c$ additionally included metabolic/lifestyle variables, including ethnicity (relates to ethnic differences in adipose tissue distribution/accumulation ${ }^{10}$ ), current cigarette smoking (yes/no), current alcohol consumption (yes/no), diabetes (yes/no), hypertension (yes/no), and hypercholesterolemia (yes/no). Model 1c was only applied to body composition measures. 
In the main analyses, building upon model $1 \mathrm{~b} / \mathrm{c}$, we tested for linear and quadratic associations between brain structure (dependent variable) and body composition, specifically (i) anthropometric measures, and (ii) regional/specific body MRI measures. We used a three-step multiple linear regression approach: model $2 a$ included linear body composition term; model $2 b$ additionally included a quadratic body composition term; and model $2 c$ additionally included metabolic/lifestyle variables. Model 2a/b extends model 1b, while model 2c extends model $1 \mathrm{c}$.

We used regression models of incremental complexity to motivate the fully adjusted models, and to explore the importance of nonlinearities in body-brain association. Separate analyses were conducted for the full sample and body MRI subsample (when applicable). For brain MRI, we additionally adjusted for intracranial volume (except cortical thickness), image quality (average Euler number), and site (when applicable). Categorical variables were included as factors, continuous variables were mean-centered.

We evaluated model residuals for normality using residual vs fitted value and Q-Q plots, leading to log-transformation of dependent variables for models showing a significant departure from normality, namely: all outcomes for sample description analyses of body composition, and CSF, lateral, and third ventricle volumes for brain MRI analyses (Figures S14-S19 presents selected illustrations). Remaining dependent variables were not log-transformed.

All statistical analyses were conducted in R (version 3·5 2; https://www.r-project.org). We used Im for the regression analyses (Note S6 presents pseudocode), and computed the partial correlation coefficients, $r$, effect size directly from the t-statistics for continuous variables and via Cohen's $d$ for categorical variables. ${ }^{18}$ We used Bonferroni correction to adjust for multiple comparison at $\alpha=0 \cdot 05$ across $\mathrm{N}$ independent tests, defined as $\mathrm{N}=(3+8)(1+17)+17=215$, which is the number of regression models from sample description and main analyses for both the full sample and body MRI subsample, counting partly overlapping models once. This resulted in a study-wide significance threshold of $p \leq \alpha / \mathrm{N}=0 \cdot 0002$. We present the overall global picture of significant findings from model $2 c$, and the range of $p$-values and $r$ effect sizes (absolute values; indicated by $|r|)$. The full results are presented in the supplemental material. 


\section{Role of funding source}

The funding source did not contribute to study design, data collection, analysis, interpretation, manuscript preparation, or decision to submit the manuscript for publication. The corresponding author (TPG) had full access to the data, and TPG, TK, LTW, and OAA had final responsibility for the decision to submit for publication.

\section{Results}

Demographic variables

The full subject sample $(n=19,330)$ included more women $(n=10,104 ; 52 \cdot 3 \%)$ than men $(\mathrm{n}=9226 ; 47 \cdot 7 \%)$, and was largely of self-identified European ancestry $(96 \cdot 8 \%)$. The age-range was 44-82 years. Compared to women, men had significantly higher age, more alcohol consumers and cigarette smokers, higher anthropometric measures (except hip circumference), and more were diagnosed with diabetes, hypercholesterolemia, and hypertension (Table 1; Figure S2). Similarly, the body MRI subsample $(n=2,703)$ included more women $(n=1,496$; $55 \cdot 4 \%)$ than men $(n=1,207 ; 44 \cdot 7 \%)$, the age-range was $46-77$ years, and men had higher age and more adverse factors than women (Table S1; Figure S3).

Primarily for descriptive purposes, the sample characteristics were further explored for ageand sex-effects on body composition and brain structure in Note S7.

\section{Brain structure and anthropometric measures}

Analyses in the full sample revealed a robust body-brain association pattern, and we observed overall negative and nonlinear associations between anthropometric body composition measures and global brain volumes, and positive associations with accumbens (Figures 1, S6S8). Using model 2c (Figure 2a), the largest negative effect sizes were observed for cerebellum gray matter, brain stem, and cortical gray matter volumes, mean surface area, and cortical thickness (Figure 2b), together with significant quadratic terms suggestive of negative and accelerating reductions for cerebellum gray matter, brain stem, and cortical gray matter volume 
and thickness (Figure 2c) with higher anthropometric measures. We also observed decreasing cortical and cerebellum white matter volumes with increasing body composition measures, with indications of accelerating differences for cerebellum white matter volume. There were positive associations between anthropometric measures and CSF, third ventricle, and lateral ventricle. Lateral ventricle also displayed positive quadratic associations suggestive of accelerated expansion. Subcortical structures exhibited mixed results. Higher anthropometric measures were negatively associated with pallidum, and hippocampus, and positively associated with amygdala, accumbens, and putamen. There were significant negative quadratic terms for thalamus, hippocampus, amygdala, and accumbens, suggestive of accelerating reductions for thalamus and hippocampus, while amygdala and accumbens increases were quadratically attenuated. The effect sizes of the quadratic terms were small $(|r| \leq 0 \cdot 06)$. Findings did not reach significance across all anthropometric measures.

These findings were robust across models $2 \mathrm{a} / \mathrm{b} / \mathrm{c}$, with some adjustment of significant levels and effect sizes, with $p$ in $\left[2 \cdot 8 \times 10^{-80}, 0 \cdot 0002\right]$, and $|r|$ in $[0 \cdot 03,0 \cdot 14]$ (Figure S20; Tables S5S7). For model $2 \mathrm{c}$, compared to models $2 \mathrm{a} / \mathrm{b}$, we observed attenuation of significance levels and effect sizes with $p$ in $\left[1.6 \times 10^{-72}, 0 \cdot 0002\right]$ and $|r|$ in $[0 \cdot 03,0 \cdot 13]$ between body and brain anthropometrics. Additionally, we here observed significant associations ( $|r|$ in [0.03, 0.07]) between self-reported diabetes, hypertension, or hypercholesterolemia diagnosis, and brain structure, including lower cortical and cerebellum white and gray matter volumes, and higher ventricular volumes. Current cigarette smoking was associated with lower mean cortical thickness, while current alcohol consumption was not significantly associated with brain structure.

\section{Brain structure, anthropometric, and body MRI measures}

Analyses in the body MRI subsample (Figure 3a) revealed significant negative associations between BMI and surface area and cortical gray matter volume, and between WHR and caudate volume. For body MRI metrics (Figure 3b), liver PDFF was negatively associated with cortical gray matter thickness/volume, while TTMV was positively associated with accumbens. The associations were similar across model $2 \mathrm{a} / \mathrm{b} / \mathrm{c}$, with anthropometric measures showing significant $p$ in $\left[3.0 \times 10^{-06}, 0 \cdot 0002\right]$, and $|r|$ in [0.07, 0.09], and regional body MRI measures showing significant $p$ in $\left[1 \cdot 3 \times 10^{-07}, 8 \cdot 9 \times 10^{-05}\right]$, and $|r|$ in $[0 \cdot 08,0 \cdot 1]$. There were fewer 
significant findings for anthropometric measures in model $2 \mathrm{c}$ compared to $2 \mathrm{a} / \mathrm{b}$ (Figures S21S22; Tables S8-S15).

\section{Discussion}

In the largest study of body-brain connections to date, we mapped normal body-brain connections in 19,330 healthy participants without secondary disease effects. We identified a comprehensive picture of small, but highly significant, effects across body composition and brain structures, linking normally varying anthropometric measures to the majority of included brain structures. Further, we examined the novel body MRI measures $(n=2,703)$ in this context. We revealed significant associations between liver fat and cortical gray matter volume and thickness, and between thigh muscle volume and accumbens volume.

For global brain measures, we observed the strongest, negative and nonlinear, associations between anthropometric body composition measures and cerebellar and cortical gray matter, and brain stem structures. These findings suggest accelerating cortical/cerebellum and brain stem reductions with increasing body composition levels, but also of attenuation at low body composition. Furthermore, we observed positive associations for CSF, lateral, and third ventricles. These findings are in line with our hypothesis, and with prior research showing lower total gray matter volume, ${ }^{5-8}$ and regional cortical and cerebellar reductions ${ }^{9}$ in obese individuals. Brain atrophy is observed at higher ages. ${ }^{11}$ Although cross-sectional evidence and small effects, our findings suggests accelerating brain atrophy also at higher adiposity levels, possibly relating to regulatory differences in brain and body lipids. Prior studies on subcortical structures and obesity are limited. Here, we showed a mixed subcortical association pattern, that were similar across anthropometric measures. Accumbens, a structure associated with motivation and reward and part of the dopamine motivation system, ${ }^{19}$ showed the strongest positive associations. The association between accumbens volume and body composition is generally in line with a previous study documenting larger accumbens volume in children with increased genetic risk for obesity, ${ }^{20}$ and supports the assumption of a critical role of brain mechanisms for reward and reinforcement learning for lifestyle and dietary choices and obesity.

Higher liver fat was associated with lower/thinner cortical gray matter volume/thickness in healthy individuals. Non-alcoholic fatty liver disease is linked with metabolic factors, show 
increasing prevalence rate similar to that of obesity, and increased mortality from cardiovascular disease, and patients benefit from weight loss lifestyle interventions. ${ }^{21}$ Our findings point towards ongoing biological processes related to liver fat that may affect brain structures, or vice versa, in healthy individuals. We may only speculate that lifestyle interventions for addressing non-alcoholic fatty liver disease may be important also for brain health.

Contrary to our expectations, we did not observe any associations between visceral adipose tissue and brain structures. Instead, we observed a largely analogous pattern across anthropometric and regional body composition measures. Effect sizes were generally stronger for anthropometric measures, which may reflect combinatorial effects of multiple factors that likely influence these measures. The novel body MRI measures may capture more specific features, but were available for less than $15 \%$ of the sample.

Earlier studies have linked vascular risk factors to brain structure. ${ }^{5,8}$ Our study further corroborates this in healthy individuals, and indicates an association between fat distribution and brain structure. Effect sizes and significance levels were attenuated when we adjusted for lifestyle factors and metabolic syndrome, suggestive of complex body-brain mechanisms. Selfreported diagnosis of hypercholesterolemia, hypertension, or diabetes - factors related to metabolic health - were associated with several brain structures, while current cigarette smoking was associated with thinner cortical thickness. Thus, cardiometabolic risk factors appear important for brain health.

The observed body-brain connections cut across several body compositions measures and brain structures, and appeared fairly global. Causal mechanisms are unknown and likely highly complex and multifactorial, and our findings could be mediated by modifiable lifestyle choices with known links to obesity ${ }^{1}$; e.g. metabolic factors could influence both somatic and brain health, impaired brain health could influence somatic health, or the effects could be reciprocal as previously implied for obesity and depression. ${ }^{2}$ Physical fitness and activity - common lifestyle interventions - are associated with reduced risk for obesity, ${ }^{1}$ may counteract a genetic predisposition for obesity, ${ }^{12}$ have neuroprotective effects on the brain, ${ }^{22}$ and have been positively related to brain structure. ${ }^{13}$ We found significant associations between thigh muscle volume and accumbens, and negative nonlinear association pattern between anthropometric measures and cortical, cerebellar gray matter, and brain stem structures. Although it is 
premature to conclude, from a public health perspective, this nonlinear association in healthy participants may imply that lifestyle interventions for normalizing body fat composition could affect biological processes related to brain function and disease.

The high degree of somatic comorbidity in mental and other brain disorders requires a better understanding of the complex biological interactions between body and brain, and of how they relate to lifestyle or environmental factors. The observed body-brain connections could be associated with obesity-related neuroinflammatory processes. ${ }^{23} \mathrm{~A}$ recent large-scale metaanalysis showed increased risk for vascular dementia - similar to other vascular conditions - in both underweight and obese individuals, ${ }^{24}$ which is interesting in light of our observed nonlinear associations between body composition and brain structure. Shared genetic underpinnings could influence both adipose tissue accumulation and brain structure, similar to the genetic overlap relating immune-related disorders, ${ }^{25} \mathrm{BMI},{ }^{26}$ or cardiovascular risk factors, ${ }^{27}$ to brain disorders. Yet other complex biological and possibly polygenic processes, lifestyle/environmental factors, and/or their combinatorial effects could influence the findings. To understand both nature and nurture of somatic comorbidities in brain disorders, and brain disorders per se, further mechanistic investigations are warranted. The findings of this study may suggest that body composition is an important confounding factor that should be considered in future case-control studies.

Small effects are common across heterogeneous psychiatric disorders and research domains, including neuroimaging and genetics, making simple underlying causal mechanisms unlikely. ${ }^{28}$ Combinatorial complex mechanisms of additive small effects are more likely, but these are not well captured by smaller studies prone to both false positive and negative findings. ${ }^{16}$ Instead, large-scale investigations are needed, where effect size convergence and increasing accuracy is obtained, ${ }^{16}$ but this is challenging to achieve. To our knowledge, this is the first study of its magnitude investigating body composition, fitness metrics, and brain structure in healthy individuals. Through such large-scale investigations we may better understand small, but normal, body-brain processes. This will provide us with a better understanding of putative interactive or confounding factors in psychiatric and other brain disorders, thereby enhancing our conceptual understanding of the complex mechanisms at play.

This study had some limitations. At the time of MRI, the available diagnostic information was self-reported. We did not investigate the cumulative or unit effect of alcohol consumption or 
cigarette smoking, nor severity of hypertension, hypercholesterolemia, or diabetes diagnosis. Liver fat was assessed from selected regions of interest and not the whole liver. The observed liver-brain associations could be influenced by alcohol consumption although this was not captured by the current study. We limited our investigations to coarser brain measures. Findings deviated somewhat from prior studies using partly overlapping samples, ${ }^{7,8,29}$ probably due to differences in inclusion/exclusion criteria, sample size, image processing, and analyses pipeline. The exploratory cross-sectional design makes it difficult to disentangle cause from effect, determine underlying body-brain mechanisms, and draw final conclusions from the findings. Further investigations of sex-specific body-brain trajectories, and of combinatorial or additive effects of body composition, additional metabolic markers, and fitness metrics on brain structure, together with links to cognition, sex, age, disease risk, lifestyle, and environmental factors are needed.

Strengths of the study include an unprecedented sample size, including $\geq 160 \%$ larger sample than prior UK biobank studies, ${ }^{7,8,29}$ that were assessed using standardized procedures and MRI protocols. ${ }^{17}$ Fully automated data cleaning, inclusion and exclusion criteria, and quality control limits chance for subjective variations or errors. We build upon confirmatory analyses of known age- and sex-related associations on both body composition ${ }^{10}$ and brain structure ${ }^{11,30}$ that largely mirrored the current knowledge, which strengthened our confidence in the reported body-brain patterns. We applied rigorous diagnosis-based exclusion criteria to capture normative body-brain associations in healthy individuals, and rigorous correction for multiple comparisons.

\section{Conclusions}

Through large-scale body-brain mapping we link normally varying body composition measures to brain structure in a healthy population. The results imply correlated effects of adipose tissue and poor metabolic health on brain structure, affecting global brain structures, brain cavities, and accumbens, at higher measured body composition - yet the causal mechanisms remain unknown. It is of vital importance to investigate the underlying complex body-brain pathways, shared mechanisms of cardiometabolic risk factors and brain disorders, and lifestyle-related modifying factors. If brain structure alterations can be linked to lifestyle-related body composition characteristics, then preventive public health interventions for normalizing 
cardiometabolic risk factors, could prevent the development of disorders of the body and the brain.

\section{Data sharing}

The UK biobank resource is open for eligible researchers upon application (http://www.ukbiobank.ac.uk/register-apply/).

\section{Code availability}

We made use of publicly available resources for processing the image data and for conducting the statistical analyses. The project R-scripts will be made publicly available upon publication.

\section{Acknowledgements}

The Research Council of Norway (\#223273; \#276082); South-Eastern Norway Regional Health Authority (\#2017112); European Union's Horizon2020 Research and Innovation Programme (CoMorMent project; Grant \#847776) \& European Research Council (ERC) StG (Grant \#802998). This work was performed on Services for sensitive data (TSD), University of Oslo, Norway, with resources provided by UNINETT Sigma2 - the National Infrastructure for High Performance Computing and Data Storage in Norway.

\section{Authors' contributions}

Study design: OAA, TPG, LTW. Data preparation and image processing: TPG, TK, LTW. Data management: TPG, TK, DvdM, OBS, AW. Analytical strategy: DA, OF, TPG, UKH, KO, TM, IES, LTW, JL, ODL, RS. Statistical analysis: DA, OF, TPG, LTW. Figures: TPG. Data interpretation: OAA, TPG, KO, IES, NES, LTW. Manuscript preparation: OAA, OF, 
TPG, TK, KO, IES, NES, LTW. Funding: OAA, LTW. All authors revised the manuscript and approved the final version.

\section{Declaration of interests}

OAA has received speaker's honorarium from Lundbeck, and is a consultant to HealthLytix. JL, ODL, and RS are employed by AMRA. The remaining authors declare no conflicts of interest.

\section{References}

1 Bhupathiraju Shilpa N., Hu Frank B. Epidemiology of Obesity and Diabetes and Their Cardiovascular Complications. Circ Res 2016; 118: 1723-35.

2 Luppino FS, Wit LM de, Bouvy PF, et al. Overweight, Obesity, and Depression: A Systematic Review and Meta-analysis of Longitudinal Studies. Arch Gen Psychiatry 2010; 67: $220-9$.

3 Thompson P, Jahanshad N, Ching CRK, et al. ENIGMA and Global Neuroscience: A Decade of Large-Scale Studies of the Brain in Health and Disease across more than 40 Countries. Accepted in Translational Psychiatry, 2020 DOI:10.31234/osf.io/qnsh7.

4 Firth J, Siddiqi N, Koyanagi A, et al. The Lancet Psychiatry Commission: a blueprint for protecting physical health in people with mental illness. Lancet Psychiatry 2019; 6: 675712.

5 Friedman JI, Tang CY, de Haas HJ, et al. Brain imaging changes associated with risk factors for cardiovascular and cerebrovascular disease in asymptomatic patients. JACC Cardiovasc Imaging 2014; 7: 1039-53.

6 Willette AA, Kapogiannis D. Does the brain shrink as the waist expands? Ageing Res Rev 2015; 20: 86-97. 
7 Hamer M, Batty GD. Association of body mass index and waist-to-hip ratio with brain structure: UK Biobank study. Neurology 2019; 92: e594-600.

8 Cox SR, Lyall DM, Ritchie SJ, et al. Associations between vascular risk factors and brain MRI indices in UK Biobank. Eur Heart J 2019; 40: 2290-300.

9 García-García I, Michaud A, Dadar M, et al. Neuroanatomical differences in obesity: meta-analytic findings and their validation in an independent dataset. Int J Obes 2005 2019; 43: 943-51.

10 Tchernof A, Després J-P. Pathophysiology of Human Visceral Obesity: An Update. Physiol Rev 2013; 93: 359-404.

11 Fjell AM, Westlye LT, Grydeland H, et al. Critical ages in the life course of the adult brain: nonlinear subcortical aging. Neurobiol Aging 2013; 34: 2239-47.

12 Goodarzi MO. Genetics of obesity: what genetic association studies have taught us about the biology of obesity and its complications. Lancet Diabetes Endocrinol 2018; 6: $223-$ 36.

13 Sexton CE, Betts JF, Demnitz N, Dawes H, Ebmeier KP, Johansen-Berg H. A systematic review of MRI studies examining the relationship between physical fitness and activity and the white matter of the ageing brain. NeuroImage 2016; 131: 81-90.

14 Holtzer R, Epstein N, Mahoney JR, Izzetoglu M, Blumen HM. Neuroimaging of Mobility in Aging: A Targeted Review. J Gerontol Ser A 2014; 69: 1375-88.

15 Linge J, Borga M, West J, et al. Body Composition Profiling in the UK Biobank Imaging Study. Obesity; 26: 1785-95.

16 Westlye LT, Alnæs D, van der Meer D, Kaufmann T, Andreassen OA. PopulationBased Mapping of Polygenic Risk for Schizophrenia on the Human Brain: New Opportunities to Capture the Dimensional Aspects of Severe Mental Disorders. Biol Psychiatry 2019; 86: 499-501.

17 Miller KL, Alfaro-Almagro F, Bangerter NK, et al. Multimodal population brain imaging in the UK Biobank prospective epidemiological study. Nat Neurosci 2016; 19: 1523- 
18 Nakagawa S, Cuthill IC. Effect size, confidence interval and statistical significance: a practical guide for biologists. Biol Rev Camb Philos Soc 2007; 82: 591-605.

19 Volkow ND, Wise RA, Baler R. The dopamine motive system: implications for drug and food addiction. Nat Rev Neurosci 2017; 18: 741-52.

20 Rapuano KM, Zieselman AL, Kelley WM, Sargent JD, Heatherton TF, GilbertDiamond D. Genetic risk for obesity predicts nucleus accumbens size and responsivity to realworld food cues. Proc Natl Acad Sci 2017; 114: 160-5.

21 Rinella ME. Nonalcoholic Fatty Liver Disease: A Systematic Review. JAMA 2015; 313: $2263-73$.

22 Tari AR, Norevik CS, Scrimgeour NR, et al. Are the neuroprotective effects of exercise training systemically mediated? Prog Cardiovasc Dis 2019; 62: 94-101.

23 Cai D. Neuroinflammation and neurodegeneration in overnutrition-induced diseases. Trends Endocrinol Metab 2013; 24: 40-7.

24 Lee CM, Woodward M, Batty GD, et al. Association of anthropometry and weight change with risk of dementia and its major subtypes: A meta-analysis consisting 2.8 million adults with 57294 cases of dementia. Obes Rev 2020; n.a.: 1-14.

25 Yokoyama JS, Wang Y, Schork AJ, et al. Association Between Genetic Traits for Immune-Mediated Diseases and Alzheimer Disease. JAMA Neurol 2016; 73: 691-7.

26 Bahrami S, Steen NE, Shadrin A, et al. Shared Genetic Loci Between Body Mass Index and Major Psychiatric Disorders: A Genome-wide Association Study. JAMA Psychiatry 2020; published online Jan 8. DOI:10.1001/jamapsychiatry.2019.4188.

27 Andreassen OA, Djurovic S, Thompson WK, et al. Improved Detection of Common Variants Associated with Schizophrenia by Leveraging Pleiotropy with Cardiovascular-Disease Risk Factors. Am J Hum Genet 2013; 92: 197-209.

28 Paulus MP, Thompson WK. The Challenges and Opportunities of Small Effects: The 
bioRxiv preprint doi: https://doi.org/10.1101/2020.02.29.970095; this version posted March 3, 2020. The copyright holder for this preprint (which was not certified by peer review) is the author/funder. All rights reserved. No reuse allowed without permission.

New Normal in Academic Psychiatry. JAMA Psychiatry 2019; 76: 353-4.

29 Dekkers IA, Jansen PR, Lamb HJ. Obesity, Brain Volume, and White Matter Microstructure at MRI: A Cross-sectional UK Biobank Study. Radiology 2019; 291: 763-71.

30 Ritchie SJ, Cox SR, Shen X, et al. Sex Differences in the Adult Human Brain: Evidence from 5216 UK Biobank Participants. Cereb Cortex N Y N 1991 2018; 28: 2959-75. 
Table 1: Demographics

\begin{tabular}{|c|c|c|c|c|}
\hline & $\begin{array}{l}\text { Men } \\
n=9226\end{array}$ & $\begin{array}{l}\text { Women } \\
\text { n=10,104 }\end{array}$ & $\begin{array}{l}\chi^{2} \text {-test } / \text { t-test } \\
/ \quad \text { Wilcoxon } \\
\text { rank-sum test }\end{array}$ & p-value \\
\hline Age $(\text { year })^{1}$ & $62.5 \pm 7.5$ & $62.1 \pm 7.2$ & 4.5 & $7.1 \times 10^{-6}$ \\
\hline European ancestry, $\mathrm{N}(\%)^{2}$ & $8891(96.4)$ & $9822(97.2)$ & 10.7 & 0.0011 \\
\hline Smoker, N $(\%)^{2,3}$ & $397(4.3)$ & $298(2.9)$ & 25.1 & $5.4 \times 10^{-7}$ \\
\hline Alcohol drinker, $\mathrm{N}(\%)^{2,3}$ & 8751 (94.9) & $9444(93.5)$ & 16.5 & $5.0 \times 10^{-5}$ \\
\hline Height $(\mathrm{cm})^{1}$ & $176.3 \pm 6.6$ & $162.7 \pm 6.2$ & 147.2 & $\mathbf{0}$ \\
\hline Weight $(\mathrm{kg})^{1}$ & $83.2 \pm 13.1$ & $68.3 \pm 12.6$ & 80.4 & $\mathbf{0}$ \\
\hline $\mathrm{BMI}^{1}$ & $26.7 \pm 3.8$ & $25.8 \pm 4.6$ & 15.5 & $4.5 \times 10^{-54}$ \\
\hline Waist circumference $(\mathrm{cm})^{1}$ & $93.4 \pm 10.3$ & $82.2 \pm 11.4$ & 71.6 & 0 \\
\hline Hip circumference $(\mathrm{cm})^{1}$ & $100.7 \pm 7.1$ & $100.7 \pm 9.6$ & 0.1 & 0.8895 \\
\hline WHR $^{1}$ & $0.9 \pm 0.1$ & $0.8 \pm 0.1$ & 120.6 & $\mathbf{0}$ \\
\hline Diabetes, $N(\%)^{2,3}$ & $169(1.8)$ & $84(0.8)$ & 36.6 & $1.5 \times 10^{-9}$ \\
\hline $\begin{array}{l}\text { Hypercholesterolemia, N } \\
(\%)^{2,3}\end{array}$ & $1178(12.8)$ & $734(7.3)$ & 163.3 & $2.2 \times 10^{-37}$ \\
\hline Hypertension, $\mathrm{N}(\%)^{2,3}$ & $1998(21.7)$ & $1531(15.2)$ & 136.3 & $1.8 \times 10^{-31}$ \\
\hline
\end{tabular}

Notes: Report mean \pm standard deviation for continuous variables, unless otherwise stated. Pvalues $<0.05$ considered significant. Abbreviations: BMI - body mass index; WHR - waist-tohip ratio.

${ }^{1}$ Welch two sample t-test.

${ }^{2} \chi^{2}$-test.

${ }^{3}$ Self-reported. 
Figure 1: Association pattern between selected brain structures and body composition measures.
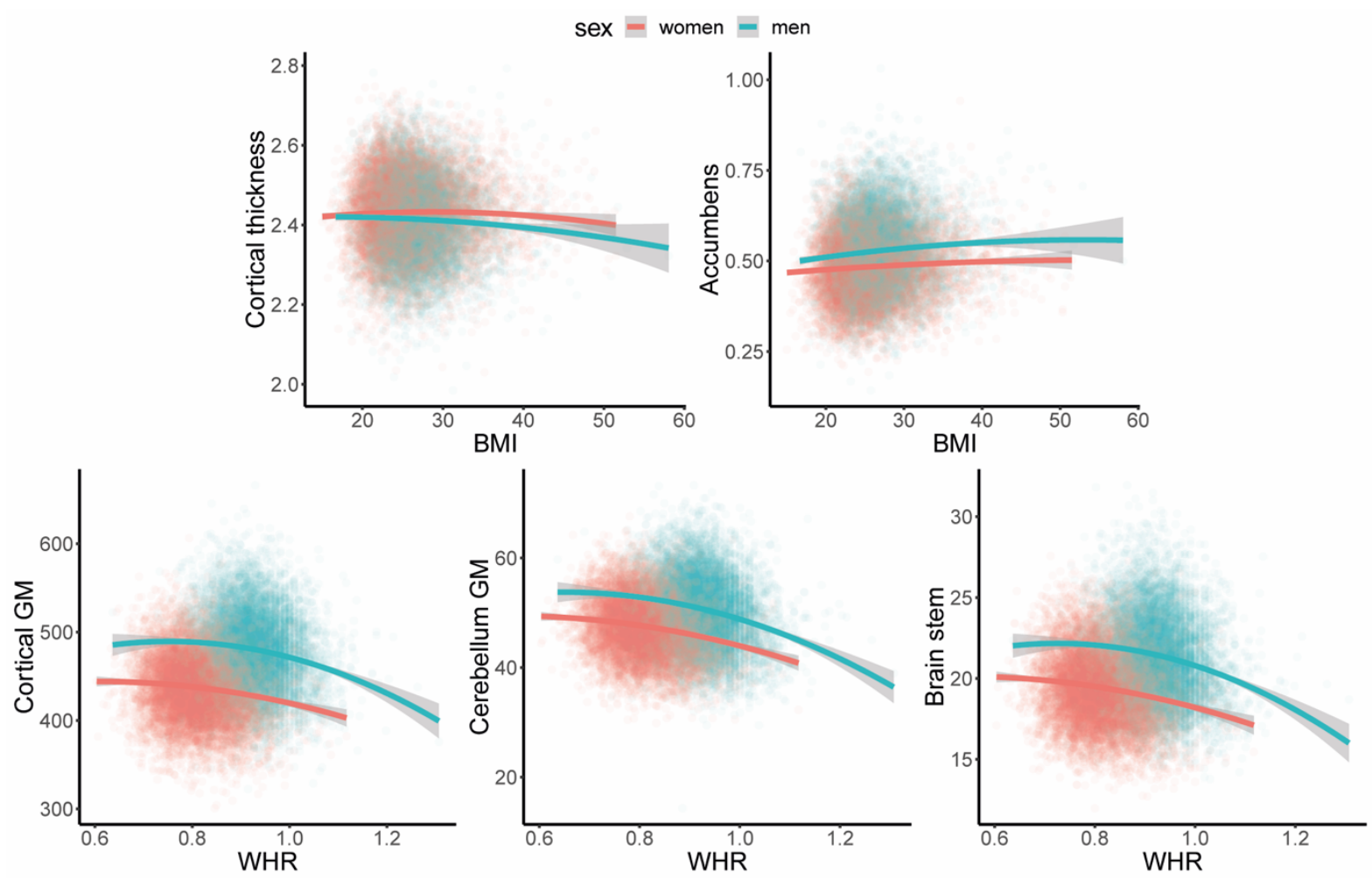

Notes: Regression lines are modeled as brain structure $=$ body composition + body composition $^{2}$. The confidence intervals are indicated in gray. Illustrations are split on sex (commonly a significant factor in neuroimaging studies), but are not adjusted for other confounders. Abbreviations: BMI - body mass index; WHR - waist-to-hip ratio. 
Figure 2: Body-brain association patterns in healthy individuals $(n=19,330)$.

a) Model for the main analysis

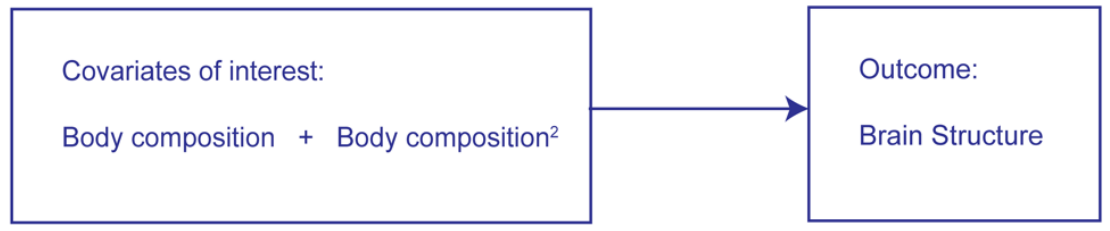

b) Effect of linear body composition term on brain structure

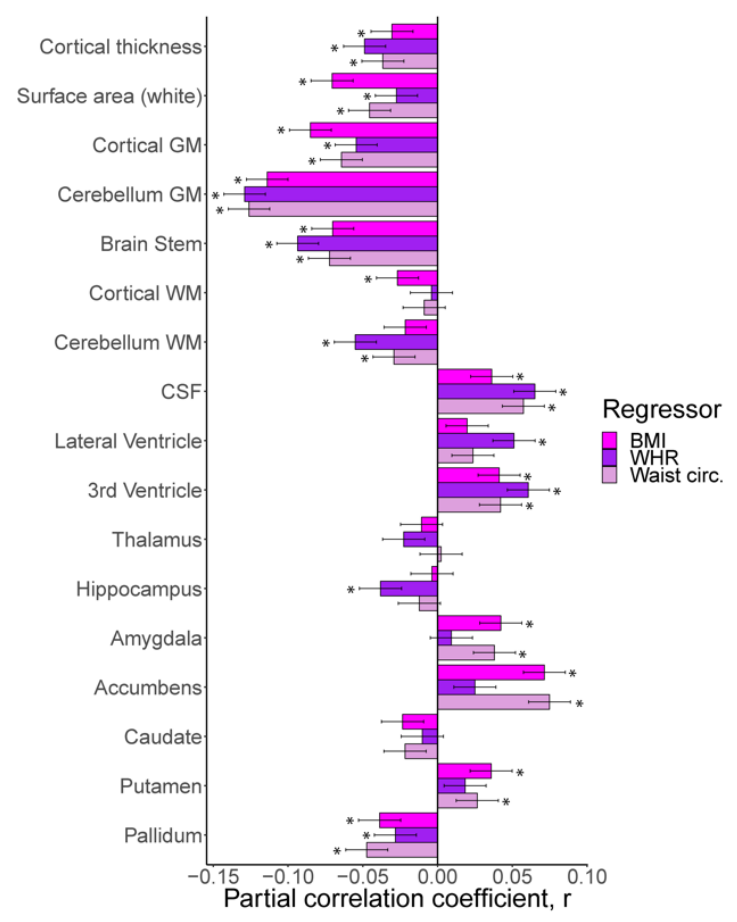

c) Effect of quadratic body composition term on brain structure

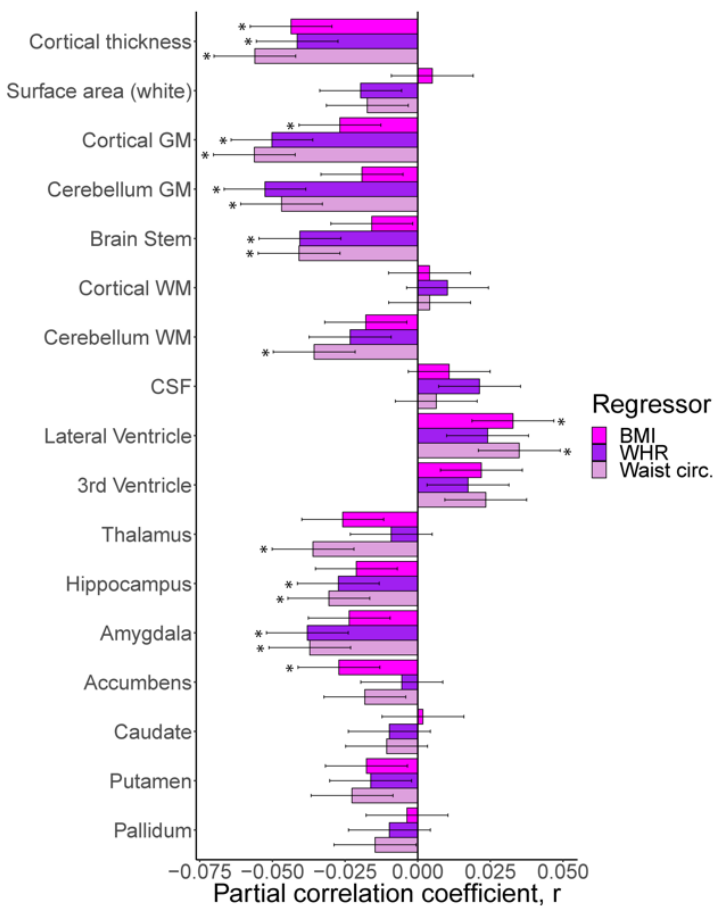

Notes: Results from model 2c (Panel a) that investigates body-brain connections through the inclusion of linear (Panel b) and quadratic (Panel c) body composition terms after adjusting for lifestyle/metabolic factors. Additional confounding variables included age, age ${ }^{2}$, sex, age-bysex, age ${ }^{2}$-by-sex, intracranial volume (except cortical thickness), Euler number, and site. Significant associations indicated by *. Dependent variables CSF, lateral $/ 3^{\text {rd }}$ ventricle were logtransformed. Abbreviations: BMI - body mass index; circ. - circumference; GM - gray matter; WHR - waist-to-hip ratio; WM - white matter. 


\section{Figure 3: Linear body-brain association pattern in healthy individuals for the MRI} subsample $(\mathbf{n}=\mathbf{2 , 7 0 3})$.

a) Effect of anthropometric measures on brain structure

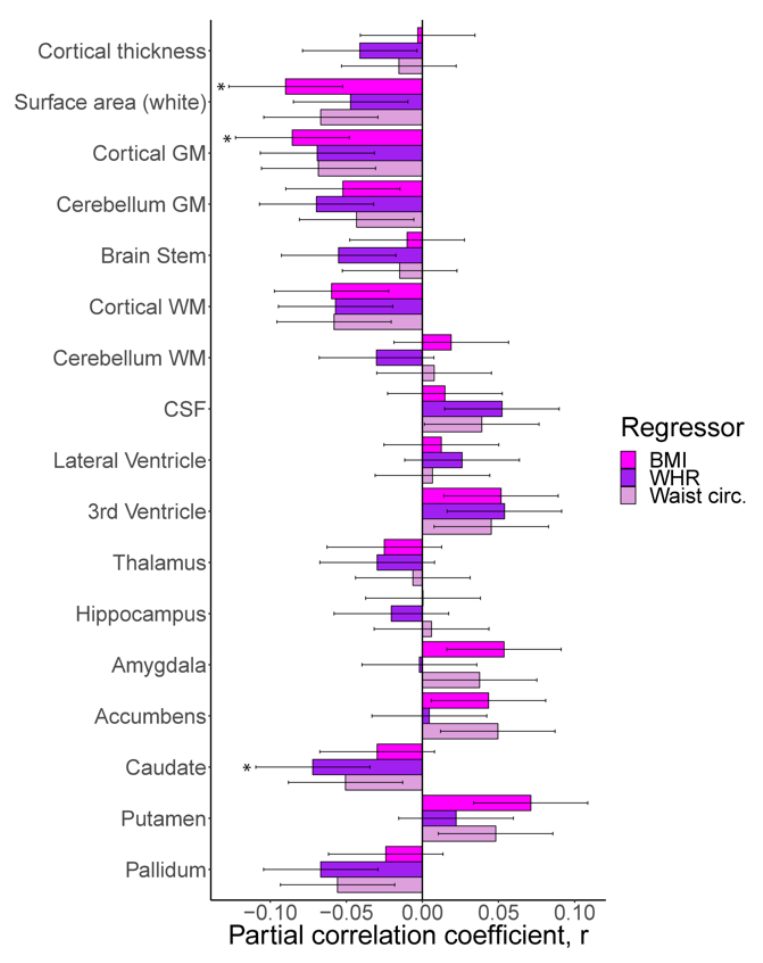

b) Effect of regional body MRI measures on brain structure

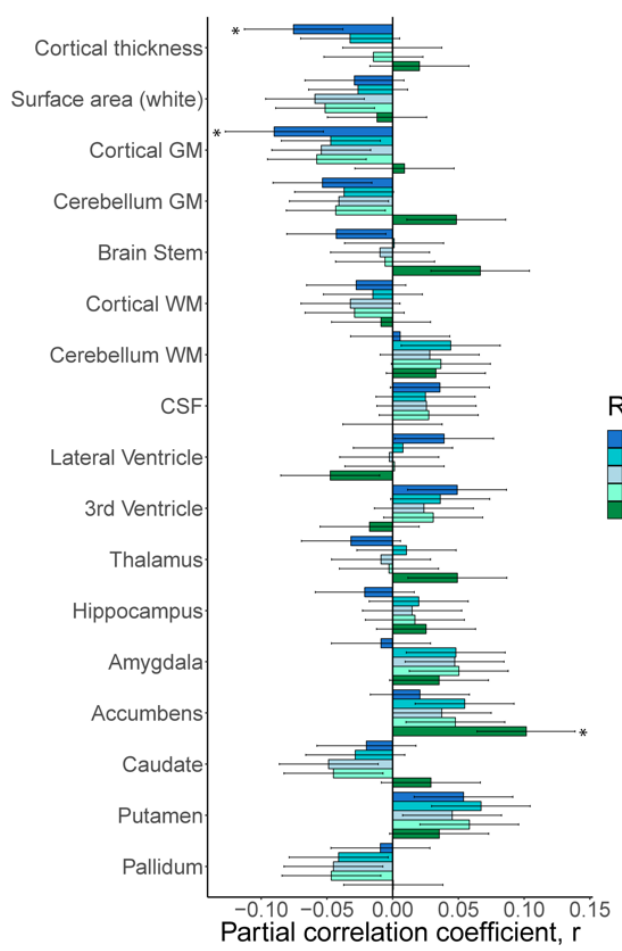

Notes: Results from model 2c that investigates body-brain connections through the inclusion of linear and quadratic body composition terms after adjusting for lifestyle/metabolic factors, for a) anthropometric body composition measures, and b) regional body MRI measures on brain structure, on brain structure. Significant associations indicated by *. Dependent variables CSF, lateral $/ 3^{\text {rd }}$ ventricle were log-transformed. Abbreviations: ASAT - abdominal subcutaneous adipose tissue; BMI - body mass index; circ. - circumference; GM- gray matter; PDFF - proton density fat fraction; TTMV - total thigh muscle volume; VAT - visceral adipose tissue; WHR - waist-to-hip ratio; $\mathrm{WM}$ - white matter. 